\title{
Inertial Range Scaling of Laminar Shear Flow as a Model of Turbulent Transport
}

\author{
Qiang Zhang ${ }^{\star}$ and James Glimm ${ }^{\star \star}$ \\ Department of Applied Mathematics and Statistics, SUNY at Stony Brook, Stony Brook, \\ NY 11794-3600, USA
}

Received April 20, 1991; in revised form November 15, 1991

Abstract. Asymptotic scaling behavior, characteristic of the inertial range, is obtained for a fractal stochastic system proposed as a model for turbulent transport.

\section{Introduction}

The determination of the statistical behavior of a fluid from the statistical properties of a random velocity field is important in the study of tracer flow in heterogeneous porous media, ground water ecology, and fully developed turbulence. In earlier work $[4,9]$, the asymptotic scaling exponents were obtained for the motion of a fluid determined by a convection-diffusion equation

$$
T_{t}+\vec{v}(\vec{x}, t) \cdot \nabla T=\mu \Delta T, \quad T(0, \vec{x})=T_{0}(\vec{x}),
$$

with $\mu=0$. Here $T$ is a physical quantity, $\vec{v}$ is a random velocity field, and $\mu$ is the molecular diffusion coefficient. The purpose of this paper is to relate these exponents to the similar but in some cases distinct exponents of [1], obtained for the equation

$$
T_{t}+v(x, t) T_{y}=\mu \Delta T, \quad T(0, x, y)=T_{0}(x, y) .
$$

Our results are either independent of cutoffs, (i.e. infrared finite) and thus are properties of the self similar, or inertial, regime, or else the cutoff cannot be removed, because the inertial range scaling behavior is infrared divergent. In the infrared divergent case, the cutoff dependence of the scaling exponents is exhibited explicitly.

* Research supported in part by the U.S. Department of Energy, contract DE-FG02-90ER25084

$\star \star$ Research supported in part by the U.S. Department of Energy, contract DE-FG02-90ER25084, the National Science Foundation, grant DMS-8901884, the Army Research Office, grant DAAL03-K-0017 
The scaling exponents depend on an arbitrary parameter which characterizes the rate of cutoff removal as the scaling limit is taken. As this parameter is taken to infinity, one obtains rapid removal of the long distance cutoff, i.e. inertial range scaling. In the infrared divergent case, the scaling exponent tends to infinity as well. We conclude that in the infrared divergent case, the inertial range scaling behavior is infinite, and any scaling relation with finite exponents has an intrinsic dependence on the cutoffs which introduce a new parameter into the asymptotic behavior. For a unique value of this parameter, we recover the results of [1]. The purpose of [1] is to study the coupling between the energy containing region and the inertial region. In [1] scaling limits occur on the same length scale as the (long distance, or small momentum) cutoff. In keeping with our inertial range scaling point of view, we do not scale the initial data while taking the asymptotic limit. Thus our scaling laws apply to all solutions of (1.2), and not just to asymptotically low wave number ones. Additional references for the study of $(1.1)$ can be traced from $[2,3,5,6]$. The relation of our work to the fractal analysis of ground water data [8] will be presented separately. For tracer flow in porous media, $T$ is the saturation value and $\vec{v}$ is determined by the random permeability field. We assume $\mu \neq 0$ and that the velocity field $v(x, t)$ in (1.2) is a translation invariant Gaussian random field with a mean value of zero.

In the infrared divergent case, we find it convenient to include a time dependence in the infrared cutoff. This convention is not required for the existence of the scaling limits and does not affect the exponents themselves, but is utilized to achieve consistency between the asymptotic scaling exponents and the scaling behavior of the asymptotic equations. The time scale in the infrared cutoff appears in a dimensionless form $t / t_{c}$. Here $t_{c}$ is a characteristic time scale, for simplicity set to one.

\section{Asymptotic Scaling Exponents}

We define the asymptotic scaling exponents of a function $F(x, y, t)$. Let $f$ and $g$ be functions which are monotonically increasing in the asymptotic regime, and let $\phi=x / f(t)$ and $\psi=y / g(t)$. Suppose that in the limit, $x, y, t \rightarrow \infty$, with $\phi$ and $\psi$ fixed, $F(x, y, t)$ approaches an asymptotic form $F^{*}(\phi, \psi) \neq 0$. We then call $f(t)$ and $g(t)$ the scaling of $x$ and $y$ in this asymptotic regime. We introduce scaled variables $x^{\prime}=\sigma x, y^{\prime}=\delta y$ and $t^{\prime}=\rho^{2} t$. Then the limit $x, y, t \rightarrow \infty$ is equivalent to the limit $\sigma, \delta, \rho \rightarrow 0$ (with $x^{\prime}, y^{\prime}$ and $t^{\prime}$ fixed). We consider $\sigma$ and $\delta$ to be functions of $\rho$. The asymptotic scaling behavior of $x$ and $y$ is determined from

$$
\gamma_{x}=\frac{1}{2} \lim _{\rho \rightarrow 0} \frac{d \ln \sigma(\rho)}{d \ln \rho} \text { and } \gamma_{y}=\frac{1}{2} \lim _{\rho \rightarrow 0} \frac{d \ln \delta(\rho)}{d \ln \rho} .
$$

In the asymptotic regime $x$ and $y$ scale as $t^{\gamma_{x}}$ and $t^{\gamma_{y}}$ respectively. When the scaling exponent equals $1 / 2$, the diffusion is normal. Any other value corresponds to anomalous diffusion.

Since (1.2) is linear and $v$ is independent of $y$, the solution of (1.2) can be expressed as

$$
T(t, x, y)=\frac{1}{2 \pi} \iint e^{i y \xi} p(t, x, \eta, \xi) \tilde{T}_{0}(\eta, \xi) d \eta d \xi
$$


where $\tilde{T}_{0}$ is the Fourier transform of $T_{0}$ and $p$ is determined by

$$
p_{t}+i \xi v(x, t) p=-\mu \xi^{2} p+\mu p_{x x}, \quad p(0, x, \eta, \xi)=e^{i x \eta} .
$$

The solution of (2.3) is given by the Feynmann-Kac formula [7]

$$
p(t, x, \eta, \xi)=\exp \left(i x \eta-\mu \xi^{2} t\right) E\left\{\exp \left[i \eta \sqrt{2 \mu} \beta(t)+i \xi \int_{0}^{t} v(x+\sqrt{2 \mu} \beta(s), t-s) d s\right]\right\},
$$

where $E\{\cdot\}$ denotes the integral over Weiner measure, and $\beta(t)$ is a Brownian path at time $t$, normalized so that $\beta(0)=0$. Here $T(t, \vec{x})$ is the solution of (1.1) for a particular realization of the random velocity $v$. Let $\langle T(t, \vec{x})\rangle$ denote the ensemble average of $T(t, \vec{x})$ over all possible realization of $v$. An exact expression for $\langle T\rangle$ can be constructed from (2.2) and (2.4) [1], which we express in terms of scaled variables, $t^{\prime}, x^{\prime}, y^{\prime}, \eta^{\prime}=\eta / \sigma$ and $\xi^{\prime}=\xi / \delta$,

$$
\langle T(t, x, y)\rangle d x d y=\left\langle T\left(\frac{t^{\prime}}{\rho^{2}}, \frac{x^{\prime}}{\sigma}, \frac{y^{\prime}}{\delta}\right)\right\rangle \sigma^{-1} \delta^{-1} d x^{\prime} d y^{\prime}=\bar{T}\left(\frac{t^{\prime}}{\rho^{2}}, \frac{x^{\prime}}{\sigma}, \frac{y^{\prime}}{\delta}\right) d x^{\prime} d y^{\prime},
$$

where

$$
\begin{aligned}
\bar{T}\left(\frac{t}{\rho^{2}}, \frac{x}{\sigma}, \frac{y}{\delta}\right)= & \left\langle T\left(\frac{t}{\rho^{2}}, \frac{x}{\sigma}, \frac{y}{\delta}\right)\right\rangle \sigma^{-1} \delta^{-1}=\frac{1}{2 \pi} \iint \exp \left(i x \eta+i y \xi-\mu \frac{\delta^{2}}{\rho^{2}} \xi^{2} t\right) \tilde{T}_{0}(\sigma \eta, \delta \xi) \\
& \cdot E\left\{\exp \left[\begin{array}{c}
\sigma \\
i-\eta \\
\rho
\end{array}\right) \sqrt{2 \mu t} \beta(1)-\frac{\xi^{2}}{2} \frac{\delta^{2} t^{2}}{\rho^{4}} \iiint_{0}^{1} R\left(\frac{\sqrt{2 \mu t}}{\rho}\left(\beta\left(s_{1}\right)-\beta\left(s_{2}\right)\right), \frac{t}{\rho^{2}}\left(s_{1}-s_{2}\right)\right)\right. \\
& \left.\left.\cdot d s_{1} d s_{2}\right]\right\} d \eta d \xi=\frac{1}{2 \pi} \iint \exp \left[i x \eta+i y \xi-\mu \xi^{2} N(\delta, \rho, t)\right] \tilde{T}_{0}(\sigma \eta, \delta \xi) \\
& \cdot E\left\{\exp \left[\begin{array}{c}
\sigma \\
i-\eta \sqrt{2 \mu t} \beta \\
\rho
\end{array}(1)-\xi^{2} A(\delta, \rho, t, \beta)\right]\right\} d \eta d \xi,
\end{aligned}
$$

$R=\langle v(x, t) v(0,0)\rangle$ is the velocity correlation function, and

$$
N(\delta, \rho, t)=\frac{\delta^{2} t}{\rho^{2}}, \quad A(\delta, \rho, t, \beta)=\frac{\delta^{2} t^{2}}{2 \rho^{4}} \int_{0}^{1} \int_{0}^{1} R\left(\frac{\sqrt{2 \mu t}}{\rho}\left(\beta\left(s_{1}\right)-\beta\left(s_{2}\right)\right), \frac{t}{\rho^{2}}\left(s_{1}-s_{2}\right)\right) d s_{1} d s_{2} .
$$

Here we have suppressed all primes on the variables $t, x, y, \eta$ and $\xi$ in (2.5) and (2.6). We will apply (2.1) to determine the scaling behavior of $\bar{T}$.

We do not scale the initial data $T_{0}$, when expressed in the original variables and when regarded as the $L_{1}$ density of a measure. Thus our initial data is

$$
T_{0}(x, y) d x d y=\sigma^{-1} \delta^{-1} T_{0}\left(\frac{x^{\prime}}{\rho}, \frac{y^{\prime}}{\delta}\right) d x^{\prime} d y^{\prime}
$$

or, dropping primes, $\sigma^{-1} \delta^{-1} T_{0}\left(\frac{x}{\sigma}, \frac{y}{\delta}\right) d x d y$. In Fourier space, the initial data is

$$
\widetilde{T}_{0}(\eta, \xi)=\widetilde{T}_{0}\left(\sigma \eta^{\prime}, \delta \xi^{\prime}\right)
$$


or, dropping primes, $\widetilde{T}_{0}(\sigma \eta, \delta \xi)$. This unscaled data, in the scaling limit, converges to a $\delta$-function in $\vec{x}$-space and to a constant in Fourier space. Alternately, data scaled in the original variables can be used as in [1], to produce unscaled data in the new variables. Except for the scaling of $T_{0}$ and the allowance of the distinct scaling behavior for $x$ and $y$ variables, (2.5) and (2.6) coincide with the formula (3.13) of [1]. $N(\delta, \rho, t)$ gives the scaling behavior due to molecular diffusion and $A(\delta, \rho, t, \beta)$ gives the scaling behavior due to the velocity correlation. Equations (2.5) and (2.6) show that $\sigma=\rho$, (i.e. $x \approx t^{1 / 2}$ ) while there are two possible scalings for $\delta$ (i.e. for $y$ ) in the asymptotic regime depending on whether or not $A(\delta, \rho, t, \cdot)$ dominates in the limit $\rho \rightarrow 0$. If $A(\delta, \rho, t, \cdot)$ dominates, then the scaling behavior of $y$ is determined by $A(\delta, \rho, t, \cdot)$ and the diffusion in the $y$ direction is anomalous. Otherwise $\delta=\rho$ $\left(y \approx t^{1 / 2}\right)$ and the diffusion is normal in the $y$ direction. The diffusion in the $x$ direction is always normal.

Lemma 2.1. If under the scaling $\delta(\rho)=\rho^{\tau}$, the limit $\lim A(\delta, \rho, t, \beta)$ exists and is finite and independent of $\beta$, then $\lim _{\rho \rightarrow 0} A(\delta, \rho, t, \beta)=\lambda t^{\tau}$, where $\lambda$ is a constant independent of $\rho, \delta, \beta$ and $t$.

Proof. From (2.6) we have

$$
A(\delta, \rho, t, \beta)=\frac{\delta^{2}}{\rho^{2 \tau}} t^{\tau}\left[\frac{1}{2}\left(\frac{t}{\rho^{2}}\right)^{2-\tau} \int_{0}^{1} \int_{0}^{1} R\left(\frac{\sqrt{2 \mu t}}{\rho}\left(\beta\left(s_{1}\right)-\beta\left(s_{2}\right)\right), \frac{t}{\rho^{2}}\left(s_{1}-s_{2}\right)\right) d s_{1} d s_{2}\right] .
$$

The expression in [ $\cdot]$ is independent of $\delta$. According to our assumption, it is finite and independent of $\beta$ in the limit $\rho \rightarrow 0$. Since $t$ and $\rho$ appear as the form of $t / \rho^{2}$ in $A,[\cdot]$ is also independent of $t$ in the limit $\rho \rightarrow 0$. Let

$$
\lambda=\lim _{\rho \rightarrow 0} \frac{1}{2}\left(\frac{t}{\rho^{2}}\right)^{2-\tau} \int_{0}^{1} \int_{0}^{1} R\left(\frac{\sqrt{2 \mu t}}{\rho}\left(\beta\left(s_{1}\right)-\beta\left(s_{2}\right)\right), \frac{t}{\rho^{2}}\left(s_{1}-s_{2}\right)\right) d s_{1} d s_{2},
$$

then the asymptotic limit of (2.7) is $\lim _{\rho \rightarrow 0} A(\delta, \rho, t, \beta)=\lambda t^{\tau}$ when $\delta=\rho^{\tau}$.

Theorem 2.2. If under the scaling $\delta(\rho)=\rho^{\tau}$ with $\tau>1,|A(\delta, \rho, t, \beta)|$ is bounded by a $\beta$-independent constant for all $\beta$, and the limit $\lim _{\rho \rightarrow 0} A(\delta, \rho, t, \beta)$ exists and is finite and independent of $\beta$, then the asymptotic form of $(2.5)$ is given by

$$
\bar{T}(t, x, y)=\lim _{\rho \rightarrow 0} \bar{T}\left(\frac{t}{\rho^{2}}, \frac{x}{\sigma}, \frac{y}{\delta}\right)=\left[4 \mu \lambda t^{1+2 \gamma_{y}}\right]^{-1 / 2} \exp \left[-\frac{x^{2}}{4 \mu t}-\frac{y^{2}}{4 \lambda t^{2 \gamma_{y}}}\right] \tilde{T}_{0}(0,0),
$$

and the asymptotic effective equation for $\bar{T}$ is given by

$$
\bar{T}_{t}=\mu \bar{T}_{x x}+2 \gamma_{y} \lambda t^{2 \gamma_{y}-1} \bar{T}_{y y}
$$

where $\lambda$ is defined by (2.8) and $\gamma_{y}=\tau / 2$ is the asymptotic exponent of $\bar{T}$ for the variable $y$.

Proof. Since $1<\tau$, we have $\lim _{\rho \rightarrow 0} N\left(\delta=\rho^{\tau}, \rho, t\right)=0 .|A(\delta, \rho, t, \beta)|$ is bounded from the assumption. From Lemma $2.1, A(\delta, \rho, t, \beta)$ converges pointwise to $\lambda t^{\tau}$ in the limit 
$\sigma \rightarrow 0$ with the scaling $\delta=\rho^{\tau}$. Therefore, applying the dominated convergence theorem, with the scaling $\sigma=\rho$ and $\delta=\rho^{\tau},(2.5)$ converges to

$$
\begin{aligned}
\bar{T}(t, x, y) & =\lim _{\rho \rightarrow 0} \bar{T}\left(\frac{t}{\rho^{2}}, \frac{x}{\sigma}, \frac{y}{\delta}\right) \\
& =\frac{1}{2 \pi} \int e^{i x \eta+i y \xi} E\left\{\exp \left[i \eta \sqrt{2 \mu t} \beta(1)-\lambda t^{\tau} \xi^{2}\right]\right\} \tilde{T}_{0}(0,0) d \eta d \xi \\
& =\frac{1}{2 \pi} \int e^{i x \eta+i y \xi} \exp \left[-\mu t \eta^{2}-\lambda t^{\tau} \xi^{2}\right] \tilde{T}_{0}(0,0) d \eta d \xi \\
& =\left[4 \mu \lambda t^{1+\tau}\right]^{-1 / 2} \exp \left[-\frac{x^{2}}{4 \mu t}-\frac{y^{2}}{4 \lambda t^{\tau}}\right] \tilde{T}_{0}(0,0) .
\end{aligned}
$$

By applying the derivative with respect to $t$ to (2.11), we obtain

$$
\bar{T}_{t}=\mu \bar{T}_{x x}+\tau \lambda t^{\tau-1} \bar{T}_{y y} \text {. }
$$

From (2.1), we have $\gamma_{x}=1 / 2$ and $\gamma_{y}=\tau / 2$. A substitution of $\tau=2 \gamma_{y}$ in (2.11) and (2.12) leads to (2.9) and (2.10).

We comment that the scalings satisfied by the asymptotic effective equation (2.12) agree with the scalings (2.1) which defines the asymptotic limits.

\section{A) The Stationary Fractal Random Velocity Field}

Theorem 2.3. Let

$$
R(x, t)=c \int_{k_{l} \leqq|k| \leqq k_{h}} e^{i k x}|k|^{1-\varepsilon} d k=2 c \int_{k_{l}}^{k_{h}} \cos (k x) k^{1-\varepsilon} d k \quad \text { for } \quad-\infty<\varepsilon<\infty,
$$

be the covariance for the fractal stationary random velocity field (cf. [1]), where $c$ and $\varepsilon$ are constants, and $k_{1}$ and $k_{h}$ are cut-offs for small and large values of $|k|$ respectively. Then the scaling exponents are given by

$$
\gamma_{x}=\frac{1}{2}, \quad \text { and } \quad \gamma_{y}=\max \left\{\frac{1}{2}, \frac{1}{2}+\frac{\varepsilon}{4}, 1+\frac{\alpha}{4}(\varepsilon-2)\right\} \quad \text { for }-\infty<\varepsilon<\infty, 1 \leqq \alpha .
$$

Here $\alpha$ characterizes the rate of infrared removal, $k_{l}=k_{0} t^{-\alpha / 2} \rho^{\alpha}$.

Remark. When Theorem 2.2 applies, we evaluate $\lambda$ in closed form.

Proof. For $\varepsilon<0$, we set $\sigma=\delta=\rho$. Then the mean field analysis given in [1] for the normal diffusion regime applies here also, Consider the regime $0<\varepsilon<2$, in which the diffusion in the $y$ direction is anomalous. In (2.5), the factor $\left.\exp \left[\begin{array}{c}i-\eta \\ \rho\end{array}\right] \sqrt{2 \mu t} \beta(1)\right]$ is bounded in magnitude and it converges to $\exp [i \eta \sqrt{2 \mu t} \beta(1)]$ with the scaling $\sigma=\rho$ for fixed $\beta$. Therefore, $\exp \left[i \underset{\rho}{i} \eta \bar{v} \sqrt{2 \mu t} \beta(1)-\xi^{2} A(\delta, \rho, t, \beta)\right]$ will converge under the 
scaling $\sigma=\rho$ and $\delta=\delta(\rho)$ if $A(\delta, \rho, t, \beta)$ converges for fixed $\beta$ under the scaling $\delta=\delta(\rho)$. The proof for the convergence and the scaling behavior of the later expression is given in [1], for $\delta=\rho^{(\varepsilon+2) / 2}$. Thus (2.5) converges under the scaling $\sigma=\rho$ and $\delta=\rho^{(\varepsilon+2) / 2}$ and from (2.1) we have proved Theorem 2.3 for $\varepsilon<2$. For $\varepsilon<2$, the infrared cut-off $k_{l}$ can be removed while for $0<\varepsilon$, the ultraviolet cut-off $k_{h}$ can be removed.

We show next that for $2 \leqq \varepsilon$, the $y$-scaling exponent $\gamma_{y}$ depends on the approach of $k_{l}$ to zero and thus is infrared divergent. Let $k_{l}$ approach zero at a rate $\rho^{\alpha}$ with $\alpha \geqq 1$. We set $k_{l}=k_{0} t^{-\alpha / 2} \rho^{\alpha}$. Here we have included the factor $t^{-\alpha / 2}$. In the infrared divergent cases, this factor is necessary to achieve the consistency between the scalings determined from $\rho, \delta$ and $\sigma$ and the scalings determined from $t, x$, and $y$ variables of the asymptotic effective equation given by (2.12). This factor does not effect the existence of the scaling limits. By changing to the variable $k^{\prime}=k t^{\alpha / 2} \rho^{-\alpha}$, we have

$$
A(\delta, \rho, t, \beta)=c \frac{\delta^{2} t^{[4+\alpha(\varepsilon-2)] / 2}}{\rho^{4+\alpha(\varepsilon-2)}} \int_{0}^{1} \int_{0}^{1} \int_{k_{0}}^{\infty} \cos \left[\sqrt{2 \mu t} t^{-\alpha / 2} \rho^{\alpha-1}\left|\beta\left(s_{1}\right)-\beta\left(s_{2}\right)\right| k^{\prime}\right] k^{\prime 1-\varepsilon} d k^{\prime} d s_{1} d s_{2} .
$$

Here we have removed the ultraviolet cutoff.

We consider $2<\varepsilon$ first, With the scaling $\delta=\rho^{(4+\alpha(\varepsilon-2)] / 2}$, we have

$$
|A(\delta, \rho, t, \beta)| \leqq c t^{[4+\alpha(\varepsilon-2)] / 2} k_{0}^{2-\varepsilon} /(\varepsilon-2),
$$

and $A(\delta, \rho, t, \beta)$ converges pointwise to

$$
\lim _{\rho \rightarrow 0} A(\delta, \rho, t, \beta)=c t^{(\varepsilon+2) / 2} \int_{0}^{1} \int_{0}^{1} \int_{k_{0}}^{\infty} \cos \left[\sqrt{2 \mu}\left|\beta\left(s_{1}\right)-\beta\left(s_{2}\right)\right| k^{\prime}\right]\left|k^{\prime}\right|^{1-\varepsilon} d k^{\prime} d s_{1} d s_{2}
$$

when $\alpha=1$ or to

$$
\lim _{\rho \rightarrow 0} A(\delta, \rho, t, \beta)=c t^{[4+\alpha(\varepsilon-2)] / 2} k_{0}^{\varepsilon-2} /(\varepsilon-2)
$$

when $1<\alpha$ (for fixed $\beta$ ) in the limit $\rho \rightarrow 0$. Applying the dominated convergence theorem, the asymptotic scaling limits of (2.5) exist and the scaling exponents are given by (2.14). Notice that $\lim _{\alpha \rightarrow \infty} \gamma_{y}=\infty$ for $2<\varepsilon$ and when $\alpha=1$ the scalings have the same functional form as for the regime $0<\varepsilon<2$.

For $\alpha=1$ the asymptotic diffusion is non-local. $1<\alpha$ the asymptotic diffusion is local. The asymptotic solution and the asymptotic effective equation are given by Theorem 2.2, (2.9) and (2.10), with $\gamma_{y}=[4+\alpha(\varepsilon-2)] / 4$ and $\lambda=c k_{0}^{2-\varepsilon} /(\varepsilon-2)$ for $2<\varepsilon$ and $1<\alpha$.

In [1], $k_{l}$ approaches to zero as $k_{l}=k_{0} \delta$ for $2<\varepsilon$. This gives the relation $\alpha=2 \gamma_{y}$. Using (2.14), we have $\gamma_{y}=\alpha / 2=2 /(4-\varepsilon)$ for $2<\varepsilon$. Therefore, we have recovered the result of [1] with this specific choice of $\alpha$. Such scaling is possible only for $\varepsilon<4$, since this choice of $\alpha$ is negative when $4<\varepsilon$.

Now we consider the case $\varepsilon=2$. In this case, (2.15) can be written as

$$
A(\delta, \rho, t, \beta)=-c \frac{\delta^{2} t^{2}}{\rho^{4}} \int_{0}^{1} \int_{0}^{1} c i\left[\sqrt{2 \mu t} t^{-\alpha / 2} \rho^{\alpha-1}\left|\beta\left(s_{1}\right)-\beta\left(s_{2}\right)\right| k_{0}\right] d s_{1} d s_{2} .
$$


Here $\operatorname{ci}(x)=-\int_{x}^{\infty} t^{-1} \cos (t) d t$ is the cosine integral and

$$
|c i(x)|=\left|C+\ln (x)-2 \int_{0}^{x / 2} t^{-1} \sin ^{2}(t) d t\right| \leqq C+1+3|\ln (x)|,
$$

where $C$ is the Euler constant. When $\alpha=1$, we choose the scaling $\delta=\rho^{2}$, then

$$
|A(\delta, \rho, t, \beta)| \leqq \bar{A}(t, \beta)=c t^{2}\left\{C+1+\frac{3}{2}\left|\ln \left(2 \mu k_{0}^{2}\right)\right|+3 \int_{0}^{1} \int_{0}^{1}\left|\ln \left(\beta\left(s_{1}\right)-\beta\left(s_{2}\right)\right)\right| d s_{1} d s_{2}\right\}
$$

and

$$
E\{\bar{A}(t, \beta)\} \leqq c t^{2}\left\{C+1+\frac{3}{2}\left|\ln \left(2 \mu k_{0}^{2}\right)\right|+3 E\left\{\int_{0}^{1} \int_{0}^{1}\left|\ln \left(\beta\left(s_{1}\right)-\beta\left(s_{2}\right)\right)\right| d s_{1} d s_{2}\right\}\right\} .
$$

Since

$$
E\left\{\int_{0}^{1} \int_{0}^{1}\left|\ln \left(\beta\left(s_{1}\right)-\beta\left(s_{2}\right)\right)\right| d s_{1} d s_{2}\right\}<\infty
$$

we have $E[\bar{A}(t, \beta)]<\infty$. For $\alpha=1$, with the scaling $\delta=\rho^{2}, A(\delta, \rho, t, \beta)$ converges pointwise to

$$
\lim _{\rho \rightarrow 0} A(\delta, \rho, t, \beta)=-c t^{2} \int_{0}^{1} \int_{0}^{1} c i\left[\sqrt{2 \mu}\left|\beta\left(s_{1}\right)-\beta\left(s_{2}\right)\right| k_{0}\right] d s_{1} d s_{2} .
$$

Applying the dominated convergence theorem, the scaling limit exists and scaling exponents satisfy (2.14).

Similarly, for $1<\alpha$, we choose $\delta=\rho^{2}|\ln \rho|^{-1 / 2}$ for $\rho \leqq \rho_{0}<1$. Then

$$
\begin{aligned}
|A(\delta, \rho, t, \beta)| \leqq \bar{A}(t, \beta)= & c t^{2}\left\{\alpha-1+\left|\ln \rho_{0}\right|^{-1}\left\{C+1+\frac{3}{2} \ln \left(2 \mu k_{0}^{2} t^{1-\alpha}\right)\right.\right. \\
& \left.\left.+3 \int_{0}^{1} \int_{0}^{1}\left|\ln \left(\beta\left(s_{1}\right)-\beta\left(s_{2}\right)\right)\right| d s_{1} d s_{2}\right\}\right\} .
\end{aligned}
$$

From (2.16), we have $E[\bar{A}(t, \beta)]<\infty$. With the scaling $\delta=\rho^{2}|\ln \rho|^{-1 / 2}, A(\delta, \rho, t, \beta)$ converges to $c t^{2}(\alpha-1)$ pointwise in the limit $\rho \rightarrow 0$. Applying the dominated convergence theorem the scaling limit exists and the scaling exponents are the same as the $\alpha=1$ case. Following the same procedure as in the proof of Theorem 2.2, one can show that the asymptotic solution and the asymptotic effective equation are given by (2.9) and (2.10) with $\gamma_{y}=1$ and $\lambda=c(\alpha-1)$ for $\varepsilon=2$ and $1<\alpha$. We have completed the proof of Theorem 2.3.

\section{B) The Time Dependent Fractal Random Velocity Field}

Theorem 2.4. Let

$$
R(x, t)=2 c \int_{k_{l}}^{k_{h}} \cos (k x) e^{-a k^{z}|t|} k^{1-\varepsilon} d k \quad \text { for }-\infty<\varepsilon<\infty, 0<z,
$$

be the covariance for the time dependent fractal random velocity field (cf. [1]), where 
$a, c, \varepsilon$ and $z$ are constants. Then the scaling exponents are $\gamma_{x}=\frac{1}{2}$ and

$$
\gamma_{y}= \begin{cases}\max \left\{\frac{1}{2}, \frac{1}{2}+\frac{\varepsilon}{4}, 1+\frac{\alpha}{4}(\varepsilon-2)\right\}, & \text { for }-\infty<\varepsilon<\infty, 2 \leqq z, 1 \leqq \alpha, \\ \max \left\{\frac{1}{2}, 1+\frac{\varepsilon-2}{2 z}\right\}, & \text { for }-\infty<\varepsilon<2,0<z<2, \\ \frac{1}{2}+\alpha \frac{\varepsilon-2}{4}+\min \left\{\frac{1}{2}, \frac{\alpha z}{4}\right\}, & \text { for } 2 \leqq \varepsilon<\infty, 0<z<2,1<\alpha .\end{cases}
$$

The parameters $k_{l}, k_{h}$ and $\alpha$ are defined in Theorem 2.3.

Remark. There are five regimes in which $\gamma_{y}$ assumes distinct functional forms, as shown in Fig. 1 and Table 1. The infrared cut-off can be removed in the regimes $R_{i}, R_{i i}$ and $R_{i i i}$ while the ultraviolet cut-off can be removed in the regimes $R_{i i}, R_{i i i}, R_{i v}$, and $R_{v}$. The scaling of $y$ in the regimes $R_{i v}$ and $R_{v}$ is infrared divergent. Thus $\gamma_{y}$ is infinite, or it depends on the rate $\alpha$ at which $k_{l}$ approaches zero. The scaling (2.18a) for $2<z$ coincides with the scaling of the stationary random velocity field given by (2.14).

Proof. Using (2.17), (2.6) becomes

$$
\begin{aligned}
A(\delta, \rho, t, \beta)= & \frac{\delta^{2} t^{2}}{\rho^{4}} c \int_{0}^{1} \int_{0}^{1} \int_{k_{l}}^{k_{h}} \cos \left(\frac{\sqrt{2 \mu t}}{\rho}\left|\beta\left(s_{1}\right)-\beta\left(s_{2}\right)\right| k\right) \\
& \cdot \exp \left[-a k^{2} \frac{t}{\rho^{2}}\left|s_{1}-s_{2}\right|\right] k^{1-\varepsilon} d k d s_{1} d s_{2} .
\end{aligned}
$$

In the regime $R_{i}$, we set $\sigma=\delta=\rho$. Then the scaling exponents in $R_{i}$ follow the

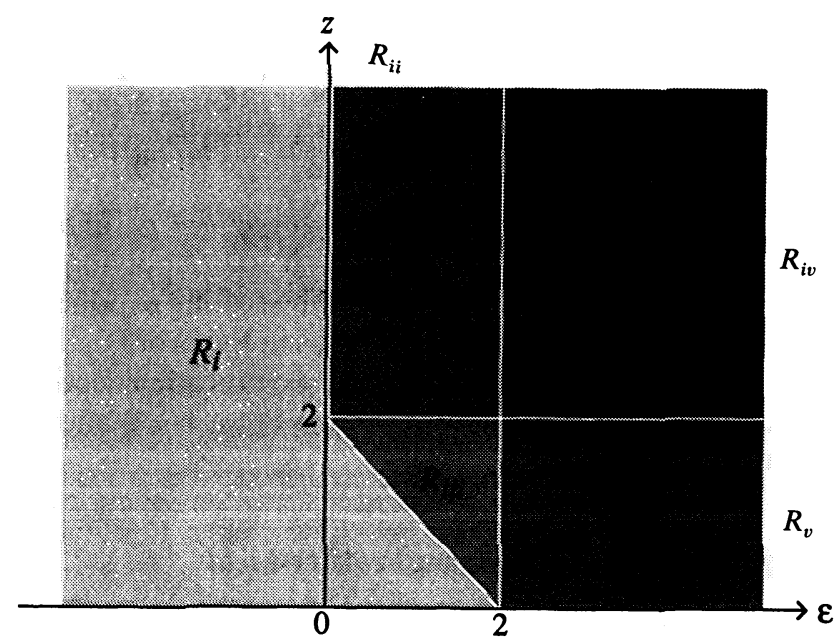

Fig. 1. Regimes for the asymptotic scaling exponent $\gamma_{y}$ of $\bar{T}$ in the $y$ direction, with the time dependent fractal velocity correlation function given by (2.17). There are five regimes in which $\gamma_{y}$ assumes distinct functional forms as indicated in the figure. The regime $R_{i}$, with $\gamma_{y}=1 / 2$, corresponds to normal diffusion, while the diffusion in the other four regimes in anomalous. The asymptotic scaling exponent of $T$ in the $x$ direction is normal, i.e. $\gamma_{x}=1 / 2$ 


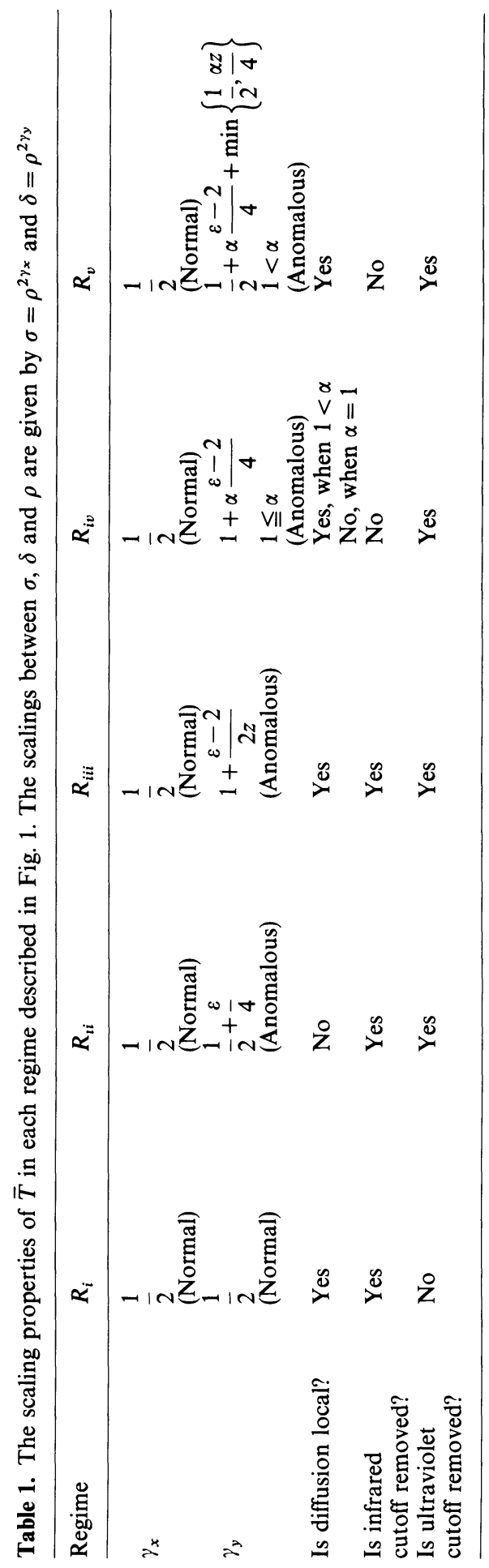


mean field analysis of [1] in this regime. The result of scaling in the regime $R_{i i}$ follow from a proof similar to the one given for the stationary random velocity field in the regime $0<\varepsilon<2$. Namely, the proof for the convergence and the scaling behavior in regime II of [1] can be applied to (2.5) in $R_{i i}$, under the scalings $\sigma=\rho$ and $\delta=\rho^{(\varepsilon+2) / 2}$, which gives $\gamma_{x}=1 / 2$ and $\gamma_{y}=(2+\varepsilon) / 4$.

For regime $R_{i i i}$, we set $k_{l}=k_{0} t^{-1 / z} \rho^{2 / z}$, and change to the variable $k^{\prime}=k t^{1 / z} \rho^{-2 / z}$. Then (2.19) can be expressed as

$$
\begin{aligned}
A(\delta, \rho, t, \beta)= & c \frac{\delta^{2} t^{(2 z+\varepsilon-2) / z}}{\rho^{(4 z+2 \varepsilon-4) / z}} \iint_{0}^{1} \int_{0}^{1} \int_{0}^{k_{h}} \cos \left[\sqrt{2 \mu t} t^{-1 / z} \rho^{(2-z) / z}\left|\beta\left(s_{1}\right)-\beta\left(s_{2}\right)\right| k^{\prime}\right] \\
& \cdot \exp \left(-a k^{\prime z}\left|s_{1}-s_{2}\right|\right) k^{\prime-\varepsilon} d k^{\prime} d s_{1} d s_{2},
\end{aligned}
$$

where $k_{h}^{\prime}=k_{h} t^{1 / z} \rho^{-2 / z}$. Since

$$
\begin{aligned}
\mid A(\delta, \rho, t, \beta) \leqq & \lim _{k_{0} \rightarrow 0 \cdot k_{h}^{\prime} \rightarrow \infty} c \frac{\delta^{2} t^{(2 z+\varepsilon-2) / z}}{\rho^{(4 z+2 \varepsilon-4) / z}} \int_{0}^{1} \int_{0}^{1} \int_{k_{0}}^{k_{h}} \exp \left(-a k^{\prime z}\left|s_{1}-s_{2}\right|\right) k^{\prime 1-\varepsilon} d k^{\prime} d s_{1} d s_{2} \\
= & \lim _{k_{0} \rightarrow 0, k_{h}^{\prime} \rightarrow \infty} c \frac{\delta^{2} t^{(2 z+\varepsilon-2) / z}}{\rho^{(4 z+2 \varepsilon-4) / z}} \int_{k_{0}}^{k_{h}} a^{-1} k^{\prime 1-\varepsilon-z} \\
& \cdot\left[1-a^{-1} k^{\prime-z}\left(1-\exp \left(-a k^{\prime z}\right)\right)\right] d k^{\prime}=\lim _{k_{0} \rightarrow 0} c \frac{\delta^{2} t^{2}(a t)^{(\varepsilon-2) / z}}{\rho^{(4 z+2 \varepsilon-4) / z}} \\
& \cdot\left[\frac{\left(a^{1 / z} k_{0}\right)^{2-\varepsilon-2 z}}{2-\varepsilon-2 z}-\frac{\left(a^{1 / z} k_{0}\right)^{2-\varepsilon-z}}{2-\varepsilon-z}+\frac{1}{z} \Gamma\left(\frac{2-\varepsilon-2 z}{z}, a k_{0}^{z}\right)\right] \\
= & \frac{\delta^{2} t^{(2 z+\varepsilon-2) / z}}{\rho^{(4 z+2 \varepsilon-4) / z}} c_{i i i}(\varepsilon, z)
\end{aligned}
$$

when $\varepsilon<2, z<2$, and $2<\varepsilon+z$, the cut-offs can be removed. Here $c_{i i i}(\varepsilon, z)=$ $c \frac{a^{(\varepsilon-2) / z}}{z} \Gamma\left(\frac{2-\varepsilon-2 z}{z}\right), \Gamma(\cdot)$ is the gamma function and $\Gamma(p, x)=\int_{x}^{\infty} t^{p-1} e^{-t} d t$ is the incomplete gamma function. The last equality of $(2.20)$ is obtained from the expansion

$$
\Gamma(p, x)=\Gamma(p)-\sum_{n=0}^{\infty}(-1)^{n} \frac{x^{p+n}}{n !(p+n)} \text { for } p \neq 0,-1,-2, \ldots
$$

We emphasize that we have removed the cut-offs before taking the limit $\rho \rightarrow 0$. Since the infrared cut-off has been removed in the regime $R_{i i i}$, the time dependent lower cutoff $k_{l}=k_{0} t^{-1 / z} \rho^{2 / z}$ is chosen for convenience rather than necessity. With the scaling $\delta=\rho^{(2 z+\varepsilon-2) / z},|A(\delta, \rho, t, \beta)|$ is bounded by $c_{i i i}(\varepsilon, z) t^{(2 z+\varepsilon-2) / z}$, and with the cutoffs removed, $A(\delta, \rho, t, \beta)$ converges pointwise to $c_{i i i}(\varepsilon, z) t^{(2 z+\varepsilon-2) / z}$, in the limit $\rho \rightarrow 0$. Therefore, from the dominated convergence theorem and Theorem 2.2 , under the scaling $\sigma=\rho$ and $\delta=\rho^{(2 z+\varepsilon-2) / z}$, the asymptotic expression for (2.5) and the asymptotic effective equation are given by (2.9) and (2.10) with

$$
\gamma_{y}=1+\frac{\varepsilon-2}{2 z} \text { and } \lambda=c_{i i i}(\varepsilon, z)=c \frac{a^{(\varepsilon-2) / z}}{z} \Gamma\left(\frac{2-\varepsilon-2 z}{z}\right)
$$

when $\varepsilon<2, z<2$, and $2<\varepsilon+z$. 
For the regimes, $R_{i v}$ and $R_{v}$, the proofs are similar to the proof for the stationary case in the regime $2<\varepsilon$. Namely we set $k_{l}=k_{0} t^{-\alpha / 2} \rho^{\alpha}$ for $1 \leqq \alpha$, and change to the variable $k^{\prime}=k t^{\alpha / 2} \rho^{-\alpha}$. Then (2.19) becomes

$$
\begin{aligned}
A(\delta, \rho, t, \beta)= & c \frac{\delta^{2} t^{[4+\alpha(\varepsilon-2)] / 2}}{\rho^{4+\alpha(\varepsilon-2)}} \int_{0}^{1} \int_{0}^{1} \int_{k_{0}}^{\infty} \cos \left[\sqrt{2 \mu t} t^{-\alpha / 2} \rho^{\alpha-1}\left|\beta\left(s_{1}\right)-\beta\left(s_{2}\right)\right| k^{\prime}\right] \\
& \cdot \exp \left[-a k^{\prime z} \rho^{\alpha z-2} t^{(2-\alpha z) / 2}\left|s-s^{\prime}\right|\right] k^{\prime 1-\varepsilon} d k^{\prime} d s_{1} d s_{2} .
\end{aligned}
$$

We have

$$
\begin{aligned}
|A(\delta, \rho, t, \beta)| \leqq & c \frac{\delta^{2} t^{[4+\alpha(\varepsilon-2)] / 2}}{\rho^{4+\alpha(\varepsilon-2)}} \int_{0}^{1} \int_{0}^{1} \int_{k_{0}}^{\infty} \exp \left[-a k^{\prime z} \rho^{\alpha z-2} t^{(2-\alpha z) / 2}\left|s-s^{\prime}\right|\right] k^{1-\varepsilon} d k^{\prime} d s_{1} d s_{2} \\
= & c \frac{\delta^{2} t^{[4+\alpha(\varepsilon-2)] / 2}}{\rho^{4+\alpha(\varepsilon-2)}} q^{-1} \int_{k_{0}}^{\infty} k^{\prime 1-\varepsilon-z}\left[1-q^{-1} k^{\prime-z}\left(1-e^{-q k^{\prime z}}\right)\right] d k^{\prime} \\
= & c \frac{\delta^{2} t^{[4+\alpha(\varepsilon-2)] / 2}}{\rho^{4+\alpha(\varepsilon-2)}} q^{(\varepsilon-2) / z}\left[-\frac{\left(q^{1 / z} k_{0}\right)^{2-\varepsilon-z}}{2-\varepsilon-z}+\frac{\left(q^{1 / z} k_{0}\right)^{2-\varepsilon-2 z}}{2-\varepsilon-2 z}\right. \\
& \left.+\frac{1}{z} \Gamma\left(\frac{2-\varepsilon-2 z}{z}, q k_{0}^{z}\right)\right] \text { for } 2<\varepsilon, \text { and } 0<z,
\end{aligned}
$$

where $q=a t^{(2-\alpha z) / 2} \rho^{\alpha z-2}$. In the limit $\rho \rightarrow 0$, there are three possible limits for $q: q \rightarrow 0, q \rightarrow a$ or $q \rightarrow \infty$ depending on the value of $\alpha$. We examine the scaling behavior of (2.22) for each of these possibilities.

When $2 / z<\alpha, \lim q=0$. Then from (2.21), (2.22) becomes

$$
|A(\delta, \rho, t, \beta)| \leqq c \frac{\delta^{2} t^{[4+\alpha(\varepsilon-2)] / 2}}{\rho^{4+\alpha(\varepsilon-2)}} \frac{k_{0}^{2-\varepsilon}}{\varepsilon-2}[1+O(q)] .
$$

Therefore, under the scaling $\delta=\rho^{(4-2 \alpha+\alpha \varepsilon) / 2},|A(\delta, \rho, t, \beta)|$ is bounded and $A(\delta, \rho, t, \beta)$ converges pointwise to

$$
\lim _{\rho \rightarrow 0} A(\delta, \rho, t, \beta)=c \frac{k_{0}^{2-\varepsilon}}{2 \varepsilon-4} t^{[4+\alpha(\varepsilon-2)] / 2} \quad \text { when } \max \left\{1, \frac{2}{z}\right\}<\alpha
$$

or to

$$
\begin{array}{r}
\lim _{\rho \rightarrow 0} A(\delta, \rho, t, \beta)=c t^{(\varepsilon+2) / 2} \iint_{000_{0}}^{1} \int_{0_{0}}^{\infty} \cos \left[\sqrt{2 \mu}\left|\beta\left(s_{1}\right)-\beta\left(s_{2}\right)\right| k^{\prime}\right] k^{\prime 1-\varepsilon} d k^{\prime} d s_{1} d s_{2} \\
\text { when } \alpha=1, z>2 .
\end{array}
$$

Therefore, applying the dominated convergence theorem, (2.5) converges under the scaling $\sigma=\rho$ and $\delta=\rho^{[4+\alpha(\varepsilon-2)] / 2}$, which gives $\gamma_{x}=1 / 2$ and $\gamma_{y}=[4+\alpha(\varepsilon-2)] / 4$ for $2 / z<\alpha$. Similarly, when $\alpha=2 / z, q=a$. Under the scaling $\delta=\rho^{(2 z+\varepsilon-2) / z}, A(\delta, \rho, t, \beta)$ is bounded and converges pointwise to

$$
\begin{aligned}
\lim _{\rho \rightarrow 0} A(\delta, \rho, t, \beta)= & c t^{2}(a t)^{(\varepsilon-2) / z}\left[-\frac{\left(a^{1 / z} k_{0}\right)^{2-\varepsilon-z}}{2-\varepsilon-z}+\frac{\left(a^{1 / z} k_{0}\right)^{2-\varepsilon-2 z}}{2-\varepsilon-2 z}\right. \\
& \left.+\frac{1}{z} \Gamma\left(\frac{2-\varepsilon-2 z}{z}, a k_{0}^{z}\right)\right] \quad \text { when } z<2
\end{aligned}
$$


or to

$$
\begin{array}{r}
\lim _{\rho \rightarrow 0} A(\delta, \rho, t, \beta)=c t^{(\varepsilon+2) / 2} \int_{00 k_{0}}^{1} \int_{0}^{\infty} \cos \left[\sqrt{2 \mu}\left|\beta\left(s_{1}\right)-\beta\left(s_{2}\right)\right| k^{\prime}\right] e^{-a k^{\prime z}} k^{\prime-\varepsilon} d k^{\prime} d s_{1} d s_{2} \\
\text { when } z=2 .
\end{array}
$$

Therefore, (2.5) converges under the scaling $\sigma=\rho$ and $\delta=\rho^{(2 z+\varepsilon-2) / z}$, and $\gamma_{x}=1 / 2$ and $\gamma_{y}=[2 z+\varepsilon-2] / 2 z$ for $\alpha=2 / z$.

Now let $\alpha<2 / z$, (this is possible only in $R_{v}$ since $1<\alpha$ ), $\lim _{\rho \rightarrow 0} q=\infty$. From the expansion

$$
\begin{array}{r}
\Gamma(p, x)=x^{p-1} e^{-x}\left[\sum_{m=0}^{M-1}(-1)^{m} \frac{\Gamma(1-p+m)}{\Gamma(1-p)} x^{-m}+O\left(x^{-M}\right)\right] \\
\text { for } x \rightarrow \infty, M=1,2, \ldots,
\end{array}
$$

(2.22) becomes

$$
|A(\delta, \rho, t, \beta)| \leqq c \frac{\delta^{2} t^{[2+\alpha(\varepsilon+z-2)] / 2}}{\rho^{2+\alpha(\varepsilon+z-2)}} \frac{a^{-1} k_{0}^{2-\varepsilon-z}}{z+\varepsilon-2}\left[1+O\left(q^{-1}\right)\right] .
$$

Therefore, under the scaling $\delta=\rho^{[2+\alpha(\varepsilon+z-2)] / 2},|A(\delta, \rho, t, \beta)|$ is bounded and $A(\delta, \rho, t, \beta)$ converges pointwise to

$$
\lim _{\rho \rightarrow 0} A(\delta, \rho, t, \beta)=c a^{-1} \frac{k_{0}^{2-\varepsilon-z}}{\varepsilon+z-2} t^{[\alpha(\varepsilon+z-2)+2] / 2} \quad \text { when } 1<\alpha<2 / z .
$$

Applying the dominated convergence theorem, the limit $\rho \rightarrow 0$ of (2.5) exists for the scaling $\sigma=\rho$ and $\delta=\rho^{[2+\alpha(\varepsilon+z-2)] / 2}$. The scaling exponents are given by $\gamma_{x}=1 / 2$ and $\gamma_{y}=[2+\alpha(\varepsilon+z-2)] / 4$ for $1<\alpha<2 / z$.

In the regime $R_{i v}$, the diffusion is non-local when $\alpha=1$ while the diffusion is local for $1<\alpha$. The diffusion in $R_{v}$ is local. From Theorem 2.2 with (2.8), (2.24), (2.25) and (2.27), we have (2.9) and (2.10) for the asymptotic solution and the asymptotic effective equation for $R_{i v}$ and $R_{v}$ with $\lambda=c k_{0}^{2-\varepsilon} /(2 \varepsilon-4)$, when $\max \{1,2 / z\}<\alpha$; and $\lambda=c a k_{0}^{2-\varepsilon-z} /(\varepsilon+z-2)$, when $1<\alpha<2 / z$; and

$$
\lambda=c a^{(\varepsilon-2) / z}\left[\frac{\left(a^{1 / z} k_{0}\right)^{(2-\varepsilon-z) / z}}{z+\varepsilon-2}-\frac{\left(a^{1 / z} k_{0}\right)^{(2-\varepsilon-2 z) / z}}{2 z+\varepsilon-2}+\frac{1}{z} \Gamma\left(\frac{2-\varepsilon-2 z}{z}, a^{1 / z} k_{0}\right)\right]
$$

when $1<\alpha=\frac{2}{z}$.

We have completed the proof of Theorem 2.4. If we set $\alpha=1(\alpha=2 / z)$ in $R_{i v}\left(R_{v}\right)$, then $\gamma_{y}$ takes the same functional form as in $R_{i i}\left(R_{i i i}\right)$.

The scalings of the local asymptotic effective equation derived here agree with scaling given by (2.18a)-(2.18c). In the limit $\alpha \rightarrow \infty, \gamma_{y} \rightarrow \infty$ also, which defines the inertial range (cut-off independent limit) scaling for regimes $R_{i v}$ and $R_{v}$.

By setting $k_{l}=k_{0} \delta$, in regimes $R_{i v}$ and $R_{v}$, we recover the scaling behavior in regime III and the portion of II with $2<\varepsilon$ of [1]. Setting $\alpha=2 \gamma_{y}$ in (2.18a) and (2.18c), we have $\gamma_{y}=\alpha / 2=2 /(4-\varepsilon)$. This scaling is only valid in the regime $4-2 z \leqq \varepsilon<4$ due to the condition $0<\alpha$ and $2 / z \leqq \alpha$. Similarly, setting $\alpha=2 \gamma_{y}$ in (2.18c), we have $\gamma_{y}=\alpha / 2=1 /(4-\varepsilon-z)$, which is only valid in the regime $\varepsilon<4-2 z$ 
due to the condition $\alpha<2 / z$. The boundary between regimes II and III of [1] for $2<\varepsilon$ is determined by $\alpha=2 / z$, i.e. $\varepsilon=4-2 z$. Our scaling in the portion with $\varepsilon<4-2 z$ of the regime $R_{i i i}$ does not agree with the scaling of the portion with $\varepsilon<2$ of the regime II of [1]. This discrepancy is due to the fact that in order to obtain inertial range scaling, we have removed the infrared cut-off in that regime. For the infrared divergent case, we obtain the inertial range scaling $\gamma_{x}=1 / 2$ and $\gamma_{y}=\infty$, and a one parameter family of finite noninertial range scalings, one of which agrees with [1].

Acknowledgements. The authors thank David H. Sharp and Ralph Menikoff for helpful comments.

\section{References}

1. Avellaneda, M., Majda, A.: Mathematical models with exact renormalization for turbulent transport. Commun. Math. Phys. 131, 381-429 (1990)

2. Collins, R. E.: Flow of fluids through porous media. Tulsa, OK: Petroleum 1976

3. Dagan, G.: Flow and transport in porous formations. Berlin, Heidelberg, New York: Springer 1989

4. Glimm, J., Sharp, D. H.: A random field model for anomalous diffusion in heterogeneous porous media. J. Stat. Phys. 62, 415-424 (1991)

5. Lake, L., Carroll, H. (eds): Reservoir characterization. New York: Academic Press 1986

6. McComb, W. D.: The physics of turbulence. Oxford: Oxford University Press 1990

7. McKean, H. P.: Stochastic integrals. New York London: Academic Press 1969

8. Neumann, S. P.: Universal scaling of hydraulic conductivities and dispersivities in geologic media. Water Resources Res. 26, 1749-1758 (1990)

9. Zhang, Q.: A multi-length scale theory of the anomalous mixing length growth for tracer flow in heterogeneous porous media. J. Stat. Phys. 66, 485-501 (1992)

Communicated by A. Jaffe 
